\title{
Numerical Treating of Mixed Integral Equation Two- Dimensional in Surface Cracks in Finite Layers of Materials
}

\author{
A. M. Al-Bugami \\ Department of Mathematics and Statistics, Collage of Sciences, Taif University, P.O. Box 11099, Taif 21944, Saudi Arabia \\ Correspondence should be addressed to A. M. Al-Bugami; abeer101aa@yahoo.com
}

Received 3 June 2021; Revised 18 July 2021; Accepted 21 December 2021; Published 25 January 2022

Academic Editor: Sergey Shmarev

Copyright (C) 2022 A. M. Al-Bugami. This is an open access article distributed under the Creative Commons Attribution License, which permits unrestricted use, distribution, and reproduction in any medium, provided the original work is properly cited.

\begin{abstract}
The goal of this paper is study the mixed integral equation with singular kernel in two-dimensional adding to the time in the Volterra integral term numerically. We established the problem from the plane strain problem for the bounded layer medium composed of different materials that contains a crack on one of the interface. Also, the existence of a unique solution of the equation proved. Therefore, a numerical method is used to translate our problem to a system of two-dimensional Fredholm integral equations (STDFIEs). Then, Toeplitz matrix (TMM) and the Nystrom product methods (NPM) are used to solve the STDFIEs with Cauchy kernel. Numerical examples are presented, and their results are compared with the analytical solution to demonstrate the validity and applicability of the methods. The codes were written in Maple.
\end{abstract}

\section{Introduction}

Many problems of engineering, mathematical physical, and contact problems in the theory of elasticity lead to singular integral equations. Integral equations provide an important tool for solving the ordinary and partial deferential equations. Therefore, many different methods are used to obtain the solution of the linear and nonlinear integral equations. Brunner and Kauthen [1] introduced collocation and iterated collocation methods for solving the two-dimensional Volterra integral equation (T-DVIE). In [2], authors proposed a class of explicit Runge-Kutta-type methods of order 3 for solving nonlinear T-DVIE. In [3], authors studied the approximate solution of T-DVIEs by the two-dimensional differential transform method. Abdou, in [4, 5], used different methods to obtain the solution of F-VIE of the first and second kinds in which the Fredholm integral term is considered in position while the Volterra integral term is considered in time. EL-Borai et al., in [6], studied the numerical solution for the T-DFIE with weak singular kernel, but they have studied the problem on a rectangular path of the parties only. AL-Bugami in [7] studied and discussed the solution of the T-DFIE with applications in contact problems. ALBugami in [8] studied and discussed the solution of the two-dimensional singular Fredholm integral equation ( $\mathrm{T}$ DFIE) with time. The solution of a large of mixed boundary value problem of a great variety of contact and crack problems in solid mechanics, physical, and engineering can be related to a system of the singular IEs have a simple Cauchy-type singularity (Ref. [9]). In [10], the authors studied the linear two-dimensional Volterra integral equation with continuous kernel numerically. In [11], the authors discussed continuous Fredholm-Volterra integral equation. AlBugami, in [12], studied the singular Hammerstein-Volterra integral equation and solved numerically. In [13], the author studied the surface cracks of finite layers of fractional materials.

In this work, we consider a mixed integral equation with singular kernel in two-dimensional (MIE)

$$
\begin{aligned}
& \eta w(x, t)+\frac{\lambda}{\pi} \int_{-1}^{1} \int_{-1}^{1} p_{1}|x-u| p_{2}|y-v| w(u, v ; t) d u d v \\
& \quad+\lambda \int_{-1}^{1} k(x, y) w(y, t) d y+\int_{0}^{t} \zeta(t, \tau) w(x, \tau) d \tau=f(x, t) .
\end{aligned}
$$

Formula (1) is called the MIE with singular kernel in 
two-dimensional of the second kind with Cauchy kernel in $\left(L_{2}(-1,1) \times L_{2}(-1,1)\right) \times C(0 ; Y) ; Y<1$, where the FI term is considered in position with singular kernel, and the VI term is considered in time with a positive and continuous kernel $\zeta(t, \tau) . f(x, t)$ is known function, while $w(x, t)$ is unknown function to be determined. The numerical coefficient $\lambda$ is called the parameter of the IE.

\section{The Basic Formulas of the Problem}

Consider the plane strain problem for the bounded layer medium (Figure 1), composed of three different materials. Let the medium material contain a crack on one of the interface. Without any loss in generality, the half length of the crack is assumed to be unity. We will consider with the effect of the ratio of the layer thickness to the crack length on the stress, intensity factors, and the strain energy release rate. For interesting the disturbed stress state, while is variable also with time, caused by the crack.

We assume that the overall stress distribution $\sigma_{i j}^{(0)}(x, y$, $t$ ), in the imperfection free medium, is known. The stress state $\sigma_{i j}^{(1)}(x, y, t)$, in the cracked medium, may be expressed as

$$
\sigma_{i j}^{(1)}(x, y, t)=\sigma_{i j}^{(0)}(x, y, t)+\sigma_{i j}(x, y, t), i, j=x, y, z,
$$

where $\sigma_{i j}$ is the disturbed state, which may be obtained by using the tractions

$$
P_{1}(x, t)=-\sigma_{y y}^{(0)}(x, 0 ; t) ; P_{2}(x, t)=-\sigma_{x y}^{(0)}(x, 0 ; t),|x|<1, t \in[0, T],
$$

which are the only external loads applied to the medium. The problem can always be expressed as the sum of a symmetric component and an antisymmetric component. The tractions are $P_{i}(x, t), i=1,2$, where

$$
P_{1}(x, t)=P_{1}(-x, t), P_{2}(x, t)=-P_{2}(-x, t),|x|<1, t \in[0, T] .
$$

The solution of the antisymmetric problem requires only a slight modification. Let $u_{i}, v_{i}$ be the $x$ and $y$ components of the displacement vector in the $i$-th materials and satisfy the filed equations in the form:

$$
\begin{aligned}
& \mu_{i} \nabla^{2} u_{i}+\left(\lambda_{i}+\mu_{i}\right) \frac{\partial}{\partial x}\left(\frac{\partial u_{i}}{\partial x}+\frac{\partial v_{i}}{\partial y}\right)=\rho \frac{d^{2} u_{i}}{d t^{2}} \\
& \mu_{i} \nabla^{2} v_{i}+\left(\lambda_{i}+\mu_{i}\right) \frac{\partial}{\partial x}\left(\frac{\partial u_{i}}{\partial x}+\frac{\partial v_{i}}{\partial y}\right)=\rho \frac{d^{2} u_{i}}{d t^{2}}
\end{aligned}
$$

Then, assume the displacement functions in the following:

$$
{ }_{i}(x, y, t)=U_{i}(x, y)+F(t)
$$

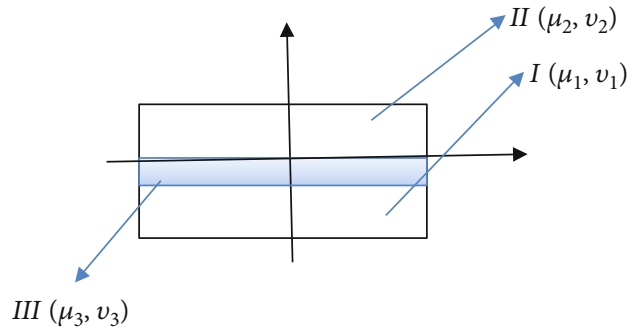

Figure 1: The layers of materials.

$$
v_{i}(x, y, t)=V_{i}(x, y)+F(t) .
$$

$F(t)$ is known function of $t$. Hence, using (7) and (8) in (5) and (6), we get

$$
\begin{gathered}
\left(\lambda_{i}+2 \mu_{i}\right) \frac{\partial^{2} U_{i}}{\partial x^{2}}+\mu_{i} \frac{\partial^{2} U_{i}}{\partial y^{2}}+\left(\lambda_{i}+\mu_{i}\right) \frac{\partial^{2} V_{i}}{\partial x \partial y}=0 \\
\left(\lambda_{i}+2 \mu_{i}\right) \frac{\partial^{2} V_{i}}{\partial y^{2}}+\mu_{i} \frac{\partial^{2} V_{i}}{\partial x^{2}}+\left(\lambda_{i}+\mu_{i}\right) \frac{\partial^{2} U_{i}}{\partial x \partial y}=0 \\
\frac{d^{2} F(t)}{d t^{2}}=\frac{\mu_{i}}{\rho} F(t) .
\end{gathered}
$$

Formula (11) has a solution

$$
F(t)=B e^{-\sqrt{\frac{\mu_{i}}{\rho}}}, F(\infty) \longrightarrow 0 .
$$

For solving the two formulas (9) and (10), we use the Fourier integral transform:

$$
\begin{gathered}
U_{i}(x, y)=\frac{2}{\pi} \int_{0}^{\infty} w_{i}(\alpha, y) \sin \alpha x d \alpha,(i=1,2,3), \\
V_{i}(x, y)=\frac{2}{\pi} \int_{0}^{\infty} \psi_{i}(\alpha, y) \cos \alpha x d \alpha .
\end{gathered}
$$

Then, we have

$$
\begin{gathered}
-\alpha^{2}\left(\lambda_{i}+2 \mu_{i}\right) w_{i}+\mu_{i} \frac{d^{2} w_{i}}{d y}-\alpha\left(\lambda_{i}+\mu_{i}\right) \frac{d \psi_{i}}{d y}=0, \\
-\alpha^{2}\left(\lambda_{i}+2 \mu_{i}\right) \psi_{i}+\mu_{i} \frac{d^{2} \psi_{i}}{d y}+\alpha\left(\lambda_{i}+\mu_{i}\right) \frac{d w_{i}}{d y}=0 .
\end{gathered}
$$

After solving the system of Eqs. (15) and (16), and then using the two formulas (13) and (14), we get

$$
\begin{gathered}
U_{i}(x, y)=\frac{2}{\pi} \int_{0}^{\infty}\left[\left(A_{i 1}+A_{i 2} y\right) e^{-\alpha y}+\left(A_{i 3}+A_{i 4} y\right) e^{\alpha y}\right] \sin \alpha x d \alpha, \\
V_{i}(x, y)=\frac{2}{\pi} \int_{0}^{\infty}\left\{\left[A_{i 1}+\left(\frac{K_{i}}{\alpha}+y\right) A_{i 1}\right] e^{-\alpha y}+\left[-A_{i 3}+\left(\frac{K_{i}}{\alpha}-y\right) A_{i 4}\right] e^{\alpha y}\right\} \cos \alpha x d \alpha,
\end{gathered}
$$

where $K_{i}$ has physical meaning and $K_{i}=3-4 v_{i}$ for plane strain and $K_{i}=\left(3-v_{i}\right) /\left(1+v_{i}\right)$ for generalized plane stress, $v_{i}$ is Poisson's coefficient for each materials, and $A_{i, j}$ 
TABLE 1: The values of exact, approximate, and absolute error values by TMM and PNM for (fiber $v=0.22$ ).

\begin{tabular}{|c|c|c|c|c|c|c|c|}
\hline$T$ & $N$ & $x$ & Exact sol. & Approx. $T$. & Error. $T$. & Approx. N. & Error. $N$. \\
\hline \multirow{12}{*}{0.02} & \multirow{6}{*}{10} & -1.00 & 0.00400000 & 0.00045299 & 0.0000529901 & 0.00445921 & 0.00045921 \\
\hline & & -0.60 & 0.00014400 & 0.00014029 & $0.370969 \times 10-{ }^{5}$ & 0.00202874 & 0.00188474 \\
\hline & & -0.20 & 0.00001600 & 0.00002022 & $0.422877 \times 10-{ }^{5}$ & 0.00264946 & 0.00263346 \\
\hline & & 0.20 & 0.00001600 & 0.00002022 & $0.422877 \times 10-{ }^{5}$ & 0.00483289 & 0.00481689 \\
\hline & & 0.60 & 0.00014400 & 0.00014029 & $0.37096 \times 10-5$ & 0.00797219 & 0.00782819 \\
\hline & & 1.00 & 0.00400000 & 0.00045299 & 0.0000529901 & 0.00246812 & 0.00153187 \\
\hline & \multirow{6}{*}{20} & -1.00 & 0.00400000 & 0.00047952 & 0.0000795200 & 0.00443268 & 0.00043268 \\
\hline & & -0.60 & 0.00014400 & 0.00014464 & $0.6491943 \times 10-{ }^{6}$ & 0.00202439 & 0.00188039 \\
\hline & & -0.20 & 0.00001600 & 0.00002348 & $0.7485778 \times 10-5$ & 0.00264620 & 0.00263020 \\
\hline & & 0.20 & 0.00001600 & 0.00002348 & $0.748577 \times 10-^{5}$ & 0.00482963 & 0.00481363 \\
\hline & & 0.60 & 0.00014400 & 0.00014464 & $0.6491944 \times 10-{ }^{6}$ & 0.00796748 & 0.00782384 \\
\hline & & 1.00 & 0.00400000 & 0.00047952 & 0.0000795200 & 0.00244159 & 0.00155840 \\
\hline \multirow{12}{*}{0.2} & \multirow{6}{*}{10} & -1.00 & 0.04000000 & 0.08709172 & 0.047091725 & 0.08217952 & 0.04217952 \\
\hline & & -0.60 & 0.01440000 & 0.02430491 & 0.009904913 & 0.02213587 & 0.00773587 \\
\hline & & -0.20 & 0.00160000 & 0.00238484 & 0.000784841 & 0.00028484 & 0.00131515 \\
\hline & & 0.20 & 0.00160000 & 0.00238484 & 0.000784841 & 0.00246827 & 0.00086827 \\
\hline & & 0.60 & 0.01440000 & 0.02430491 & 0.009904913 & 0.01619242 & 0.00179242 \\
\hline & & 1.00 & 0.04000000 & 0.08709172 & 0.047091725 & 0.08417060 & 0.04417060 \\
\hline & \multirow{6}{*}{20} & -1.00 & 0.04000000 & 0.09251755 & 0.05251755 & 0.08760535 & 0.04760535 \\
\hline & & -0.60 & 0.01440000 & 0.02518075 & 0.01078075 & 0.02301171 & 0.00861171 \\
\hline & & -0.20 & 0.00160000 & 0.00302813 & 0.00142813 & 0.00035844 & 0.00124155 \\
\hline & & 0.20 & 0.00160000 & 0.00302813 & 0.00142813 & 0.00182498 & 0.00022498 \\
\hline & & 0.60 & 0.01440000 & 0.02518075 & 0.01078075 & 0.01706826 & 0.00266826 \\
\hline & & 1.00 & 0.04000000 & 0.09251755 & 0.05251755 & 0.08959643 & 0.04959643 \\
\hline
\end{tabular}

, $j=1,2,3,4$ are functions of $\alpha$ which can be determined from the boundary conditions. After obtaining, the values of $U_{i}, V_{i}$, the stresses may be evaluated by Hooks law.

The components of the stress vector at the interfaces and boundaries may be expressed as

$$
\begin{aligned}
\frac{1}{2 \mu_{i}} \sigma_{y y}^{i}= & \frac{2}{\pi} \int_{0}^{\infty}\left\{-\left[\alpha\left(A_{i 1}+A_{i 2} y\right)+2\left(1-v_{i}\right) A_{i 2}\right] e^{-\alpha y}\right. \\
& \left.+\left[-\alpha\left(A_{i 3}+A_{i 4} y\right)+2\left(1-v_{i}\right) A_{i 4} e^{\alpha y}\right]\right\} \cos \alpha x d \alpha, \\
\frac{1}{2 \mu_{i}} \sigma_{x y}^{i}= & \frac{2}{\pi} \int_{0}^{\infty}\left\{-\left[\alpha\left(A_{i 1}+A_{i 2} y\right)+\left(1-v_{i}\right) A_{i 2}\right] e^{-\alpha y}\right. \\
& \left.+\left[\alpha\left(A_{i 3}+A_{i 4} y\right)-\left(1-2 v_{i}\right) A_{i 4}\right] e^{\alpha y}\right\} \sin \alpha x d \alpha .
\end{aligned}
$$

On the boundaries, the medium may have formally any one of the following four groups of homogeneous boundary conditions

$$
\begin{gathered}
\sigma_{y y}^{i}=0=\sigma_{x y}^{i}, \quad u_{i}=0=v_{i} \\
\sigma_{x y}^{i}=0=v_{i}, \quad \sigma_{y y}^{i}=0=u_{i}, \quad i=1,2,3 .
\end{gathered}
$$

The continuity requires that on the interfaces, the stress and displacement vectors in the adjacent layers be equal, i.e.,

$$
\begin{gathered}
u_{i+1}-u_{i}=0, \quad \mathrm{v}_{i+1}-v_{i}=0, \\
\sigma_{y y}^{i+1}-\sigma_{y y}^{i}=0, \quad \sigma_{x y}^{i+1}-\sigma_{x y}^{i}=0, \quad i=1,2,3 .
\end{gathered}
$$

Now, to obtain the integral equation, we first assume that at $y=0$, the bond between the two adjacent layers is perfect except for the dislocations at $y=0$ and $x=y$ defied by

$$
\frac{\partial}{\partial x}\left(u_{2}^{+}-u_{3}^{-}\right)=f_{1}(x, t), \frac{\partial}{\partial x}\left(v_{2}^{+}-v_{3}^{-}\right)=f_{2}(x, t),
$$

where the superscripts + and - refer to the limiting values of the displacement as $y$ approaches zero from + and - sides, respectively. In addition to (21), on the interface $y=0$, we have the following conditions

$$
\sigma_{y y}^{2}-\sigma_{y y}^{3}=0, \quad \sigma_{x y}^{2}-\sigma_{x y}^{3}=0, \quad(0 \leq x<\infty, y=0) .
$$

The components of the stress vector at $y=0$ and $x>0$ 
TABLE 2: The values of exact, approximate, and absolute error values by TMM and PNM for (Plutonium $v=0.21$ ).

\begin{tabular}{|c|c|c|c|c|c|c|c|}
\hline$T$ & $N$ & $x$ & Exact sol. & Approx. $T$. & Error $T$. & Approx. $N$. & Error $N$. \\
\hline \multirow{12}{*}{0.02} & \multirow{6}{*}{10} & -1.00 & 0.00400000 & 0.000443078 & 0.000043078 & 0.00446912 & 0.00046912 \\
\hline & & -0.60 & 0.00014400 & 0.000140836 & $0.316366 \times 10-^{5}$ & 0.00202819 & 0.00188419 \\
\hline & & -0.20 & 0.00001600 & 0.000020081 & $0.408164 \times 10-{ }^{5}$ & 0.00264960 & 0.00263360 \\
\hline & & 0.20 & 0.00001600 & 0.000020081 & $0.408164 \times 10-^{5}$ & 0.00483303 & 0.00481703 \\
\hline & & 0.60 & 0.00014400 & 0.000140836 & $0.316366 \times 10-5$ & 0.00797164 & 0.00782764 \\
\hline & & 1.00 & 0.00400000 & 0.000443078 & 0.0000430784 & 0.00247804 & 0.00152196 \\
\hline & \multirow{6}{*}{20} & -1.00 & 0.00400000 & 0.00046333 & 0.000063339 & 0.00444887 & 0.00044887 \\
\hline & & -0.60 & 0.00014400 & 0.00014484 & $0.843735 \times 10-{ }^{6}$ & 0.00202419 & 0.00188019 \\
\hline & & -0.20 & 0.00001600 & 0.00002309 & $0.7095783 \times 10-5$ & 0.00264659 & 0.00263059 \\
\hline & & 0.20 & 0.00001600 & 0.00002309 & $0.7095783 \times 10-5$ & 0.00483002 & 0.00481402 \\
\hline & & 0.60 & 0.00014400 & 0.00014484 & $0.843735 \times 10-{ }^{6}$ & 0.00796764 & 0.00782364 \\
\hline & & 1.00 & 0.00400000 & 0.00046333 & 0.000063339 & 0.00245778 & 0.00154221 \\
\hline \multirow{12}{*}{0.2} & \multirow{6}{*}{10} & -1.00 & 0.04000000 & 0.07863996 & 0.038639964 & 0.07372776 & 0.03372776 \\
\hline & & -0.60 & 0.01440000 & 0.02389663 & 0.009466379 & 0.02172759 & 0.00732759 \\
\hline & & -0.20 & 0.00160000 & 0.00258811 & 0.000988111 & 0.00008157 & 0.00151842 \\
\hline & & 0.20 & 0.00160000 & 0.00258811 & 0.000988111 & 0.00226500 & 0.00066500 \\
\hline & & 0.60 & 0.01440000 & 0.02389663 & 0.009496637 & 0.01578414 & 0.00138414 \\
\hline & & 1.00 & 0.04000000 & 0.07863996 & 0.038639964 & 0.07571884 & 0.03571884 \\
\hline & \multirow{6}{*}{20} & -1.00 & 0.04000000 & 0.08232299 & 0.04232299 & 0.07741079 & 0.03741079 \\
\hline & & -0.60 & 0.01440000 & 0.02462094 & 0.01022094 & 0.02245190 & 0.00805190 \\
\hline & & -0.20 & 0.00160000 & 0.00312893 & 0.001528933 & 0.00045924 & 0.00114075 \\
\hline & & 0.20 & 0.00160000 & 0.00312894 & 0.001528933 & 0.00172417 & 0.00012417 \\
\hline & & 0.60 & 0.01440000 & 0.02462094 & 0.010220943 & 0.01650845 & 0.00210845 \\
\hline & & 1.00 & 0.04000000 & 0.08232299 & 0.042322994 & 0.07940187 & 0.03940187 \\
\hline
\end{tabular}

may be expresses as

$$
\begin{aligned}
\frac{1+K_{3}}{\mu_{3}} \sigma_{y y}^{3}(x, 0, t)= & \lim _{y \longrightarrow 0^{-}} \frac{2}{\pi} \int_{0}^{\infty} e^{\alpha x}\left\{\alpha_{11} A_{1}(\alpha, t)+\alpha_{12} A_{2}(\alpha, t)\right\} \cos \alpha x d \alpha \\
& +\frac{2}{\pi} \int_{0}^{\infty}\left\{H_{11}(\alpha) A_{1}(\alpha, t)+H_{12}(\alpha) A_{2}(\alpha, t)\right\} \cos \alpha x d \alpha \\
& +\int_{0}^{t} F(\tau) f_{i}(x, 0, \tau) d \tau, \\
-\frac{1+K_{3}}{\mu_{3}} \sigma_{x y}^{3}(x, 0, t)= & \lim _{y \longrightarrow 0^{-}} \frac{2}{\pi} \int_{0}^{\infty} e^{\alpha x}\left\{\alpha_{21} A_{1}(\alpha, t)+\alpha_{22} A_{2}(\alpha, t)\right\} \sin \alpha x d \alpha \\
& +\frac{2}{\pi} \int_{0}^{\infty}\left\{H_{21}(\alpha) A_{1}(\alpha, t)+H_{22}(\alpha) A_{2}(\alpha, t)\right\} \sin \alpha x d \alpha \\
& +\int_{0}^{t} F(\tau) f_{i}(x, 0, \tau) d \tau,
\end{aligned}
$$

where $H(\alpha)$ is the Heaviside functions, and $A_{i}$ is the Fourier transforms of $f_{i}$ defined as follows:

$$
A_{1}(\alpha, t)=\int_{0}^{\infty} f_{1}(z, t) \cos \alpha z d z, \quad A_{2}(\alpha, t)=\int_{0}^{\infty} f_{2}(z, t) \sin \alpha z d z .
$$

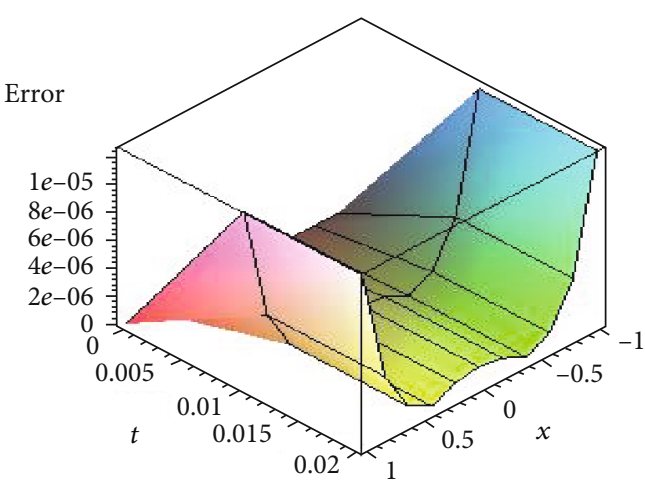

Fibber $v=0.22$

FIgure 2: The value absolute error by TMM at $N=10$ and $T=0.02$

The constants $a_{i j}$ depend on the elastic properties of the materials adjacent to the crack only and are given by

$$
\begin{aligned}
& a_{11}=-a_{22}=\left(1+\lambda_{2} \lambda_{4}\right) / \lambda_{4}, \quad a_{12}=-a_{21}=-\left(1+2 \lambda_{4}-\lambda_{2} \lambda_{4}\right) / \lambda_{4}, \\
& \lambda_{2}=\left(K_{2} \mu_{3}-K_{3} \mu_{2}\right) /\left(\mu_{2}+K_{2} \mu_{3}\right), \quad \lambda_{4}=\left(\mu_{3}+\mu_{2} K_{3}\right) /\left(\mu_{2}+\mu_{3}\right),
\end{aligned}
$$




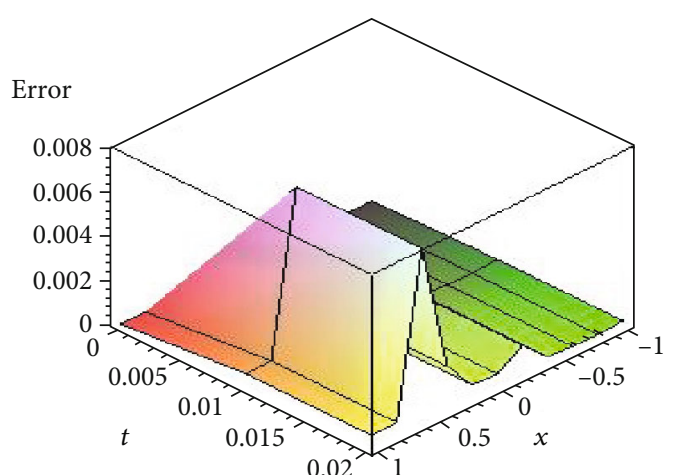

Fibber $v=0.22$

Figure 3: The value absolute error by PNM at $N=10$ and $T=0.02$.

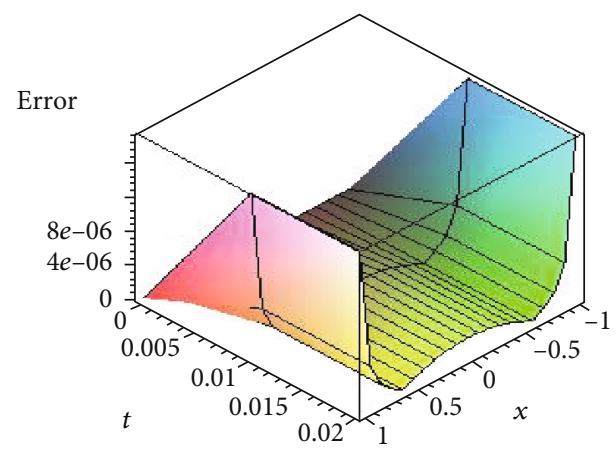

Fibber $v=0.22$

Figure 4: The value absolute error by TMM at $N=20$ and $T=0.02$

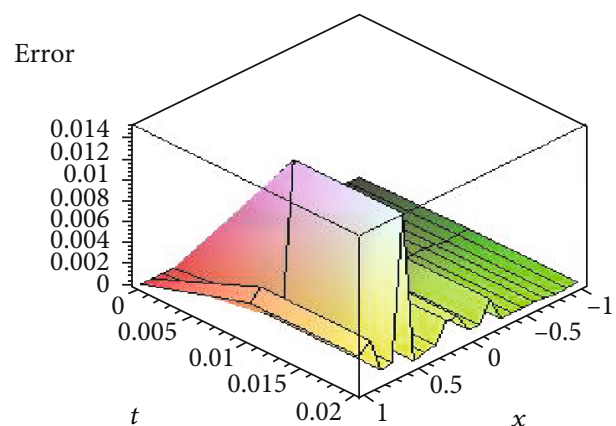

Fibber $v=0.22$

Figure 5: The value absolute error by PNM at $N=20$ and $T=0.02$.

where $\mu_{i}$ is the shear modules, and $\lambda$ 's is Lame's constants.

Note that once the dislocations $f_{i}(x)$ on the interface are specified, formulas (23)and (24) give the stresses for all values of $x$. The crack problem under consideration $f_{i}(x)$ is zero for $|x|>1$ and is unknown for $|x|<1$. On the other hand, the stress vector on the interface $y=0$ is unknown for $|x|>1$ that is given by the following known functions

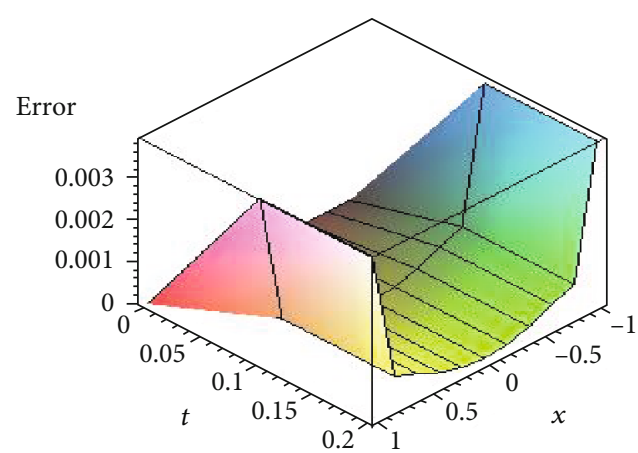

Fibber $v=0.22$

FIgURE 6: The value absolute error by TMM at $N=10$ and $T=0.2$.

for $|x|<1$, i.e.,

$$
f_{1}(x, t)=f_{1}(-x, t), f_{2}(x, t)=-f_{2}(-x, t) .
$$

Then,

$$
\begin{aligned}
\int_{0}^{\infty} & H(\alpha) \cos \alpha x d \alpha \int_{0}^{t} f_{1}(z, t) \cos \alpha z d z \\
& =\frac{1}{2} \int_{-1}^{1} \int_{-1}^{1} f_{1}^{*}(z, t) f_{1}^{* *}(z, t) d x d x \int_{0}^{\infty} H(\alpha) \cos \alpha(z-x) d \alpha .
\end{aligned}
$$

Hence, we obtain

$$
\begin{aligned}
-\frac{1+K_{3}}{\mu_{3}} P_{1}(x, t)= & \lim _{y \longrightarrow 0^{-}}\left[\frac{a_{11}}{\pi} \int_{-1}^{1} \int_{-1}^{1} f_{1}^{*}(z, t) f_{1}^{* *}(z, t) d z d z \int_{0}^{\infty} e^{\alpha y} \cos \alpha(z-x) d \alpha\right. \\
& +\frac{a_{12}}{\pi} \int_{-1}^{1} \int_{-1}^{1} f_{2}^{*}(z, t) f_{2}^{* *}(z, t) d z d z \times \int_{0}^{\infty} e^{\alpha y} \sin \alpha(z-x) d \alpha \\
& +\frac{1}{\pi} \int_{-1}^{1} \int_{-1}^{1} \sum_{j=1}^{2} k_{1 j}^{*}(x, z) k_{1 j}^{* *}(x, z) f_{j}^{*}(z, t) f_{j}^{* *}(z, t) d z d z \\
& \left.+\int_{0}^{t} F(\tau) f_{1}(x, \tau) d \tau\right],
\end{aligned}
$$

$$
\begin{aligned}
-\frac{1+K_{3}}{\mu_{3}} P_{3}(x, t)= & \lim _{y \rightarrow 0^{-}}\left[\frac{a_{21}}{\pi} \int_{-1}^{1} \int_{-1}^{1} f_{1}^{*}(z, t) f_{1}^{* * *}(z, t) d z d z \int_{0}^{\infty} e^{\alpha y} \sin \alpha(z-x) d \alpha\right. \\
& +\frac{a_{22}}{\pi} \int_{-1}^{1} \int_{-1}^{1} f_{2}^{*}(z, t) f_{2}^{* *}(z, t) d z d z \times \int_{0}^{\infty} e^{\alpha y} \cos \alpha(z-x) d \alpha \\
& \left.+\frac{1}{\pi} \int_{-1}^{1} \sum_{j=1}^{2} k_{2 j}(x, z) f_{j}(z, t) d z+\int_{0}^{t} F(\tau) f_{2}(x, \tau) d \tau\right] .
\end{aligned}
$$

Evaluating the infinite integrals in (30), passing to the 


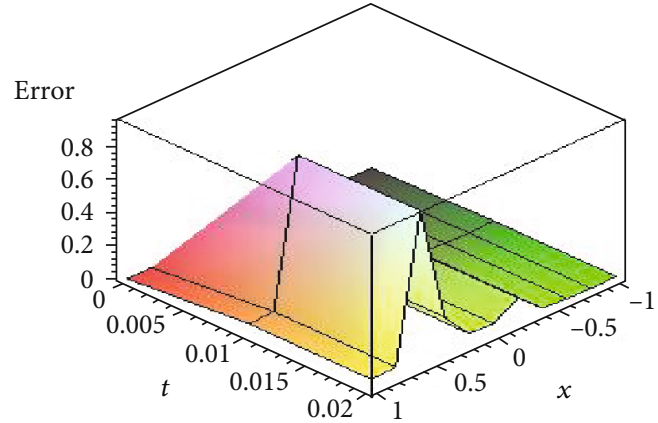

Fibber $v=0.22$

Figure 7: The value absolute error by PNM at $N=10$ and $T=0.2$.

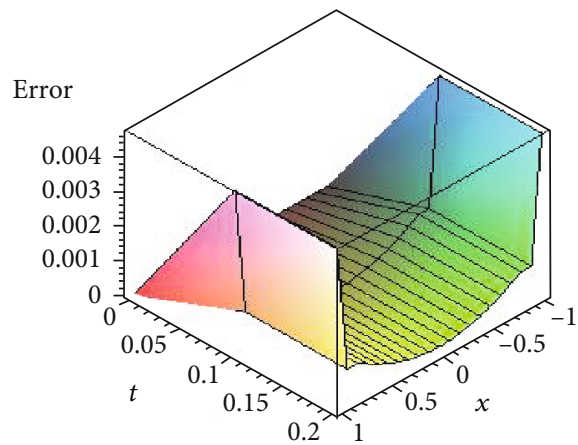

Fibber $v=0.22$

FIgure 8: The value absolute error by TMM at $N=20$ and $T=0.2$.

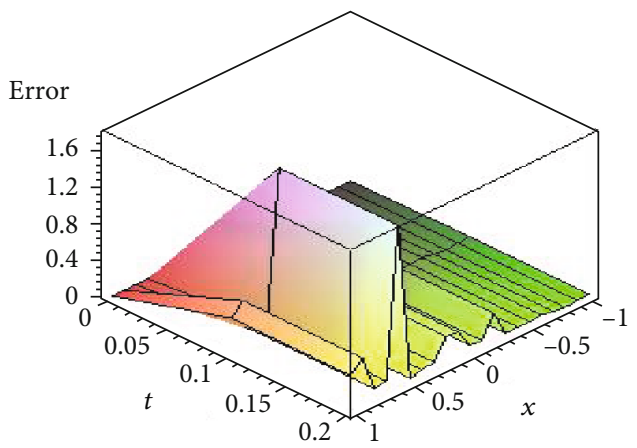

Fibber $v=0.22$

Figure 9: The value absolute error by PNM at $N=20$ and $T=0.2$.

Cauchy theorems, we have

$$
\begin{aligned}
-\frac{1+K_{3}}{a_{12} \mu_{3}} P_{1}(x, t)= & \gamma w_{1}(x, t)+\frac{1}{\pi} \int_{-1}^{1} \int_{-1}^{1} \frac{w_{2}(u, v ; t)}{(x-u)(y-v)} d u d v \\
& -\frac{1}{a_{12} \pi} \int_{-1}^{1} \sum_{j=1}^{2} k_{1 j}(x, y) w_{j}(y, t) d y+\frac{1}{a_{12}} \int_{0}^{t} F(\tau) w_{1}(x, \tau) d t, \\
& -\frac{1+K_{3}}{a_{21} \mu_{3}} P_{2}(x, t)=\frac{1}{\pi} \int_{-1}^{1} \int_{-1}^{1} \frac{w_{1}(u, v ; t)}{(x-u)(y-v)} d u d v \\
& -\gamma w_{2}(x, t)-\frac{1}{a_{21} \pi} \int_{-1}^{1} \sum_{j=1}^{2} k_{2 j}(x, y) w_{j}(y, t) d y+\frac{1}{a_{21}} \int_{0}^{t} F(\tau) w_{2}(x, \tau) d t,
\end{aligned}
$$

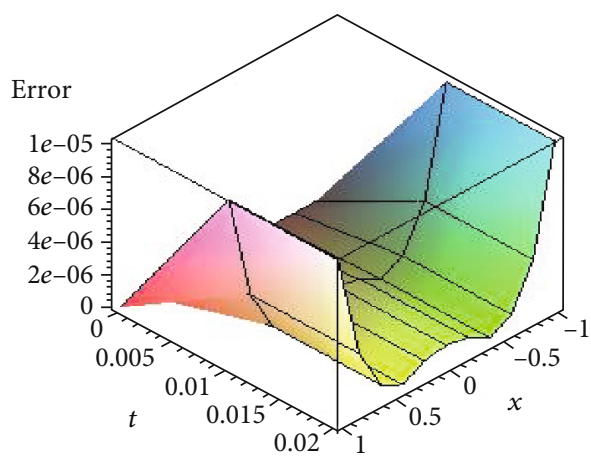

Plutonium $v=0.21$

Figure 10: The value absolute error by TMM at $N=10$ and $T=$ 0.02 .

where

$$
\gamma=\frac{a_{11}}{a_{12}}=\frac{a_{22}}{a_{21}}=\frac{\left(\mu_{2}+K_{3} \mu_{2}\right)-\left(\mu_{2}+K_{2} \mu_{3}\right)}{\left(\mu_{2}+K_{2} \mu_{3}\right)+\left(\mu_{3}+K_{3} \mu_{2}\right)} .
$$

The two formulas of (31) represent a system of MIE with Cauchy kernel. For one layer, we can have the following MIE, on noting the difference notations.

$$
\begin{gathered}
\eta w(x, t)-\frac{1}{\pi} \int_{-1}^{1} \int_{-1}^{1} \frac{w(u, v ; t)}{(x-u)(y-v)} d u d v-\frac{1}{\pi a_{12}} \int_{-1}^{1} k(x, y) w(y, t) d y \\
+\frac{1}{a_{12}} \int_{0}^{t} F(t, \tau) w(x, \tau) d \tau=f(x, t) .
\end{gathered}
$$

In general, we can write Eq. (33) in the form:

$$
\begin{gathered}
\eta w(x, t)+\frac{\lambda}{\pi} \int_{-1}^{1} \int_{-1}^{1} p_{1}|x-u| p_{2}|y-v| w(u, v ; t) d u d v \\
+\lambda \int_{-1}^{1} k(x, y) w(y, t) d y+\lambda \int_{0}^{t} \zeta(t, \tau) w(x, \tau) d \tau=f(x, t) \\
\text { where } p_{1}|x-u| p_{2}|y-v|=\frac{1}{(x-u)(y-v)}
\end{gathered}
$$

\section{The Existence and Uniqueness of the Solution}

We write this formula in the integral operator form

$$
\bar{W} w(x, t)=\frac{1}{\eta} f(x, t)-W w(x, t),(\eta \neq 0),
$$

$$
W w=H w+D w+\zeta w
$$




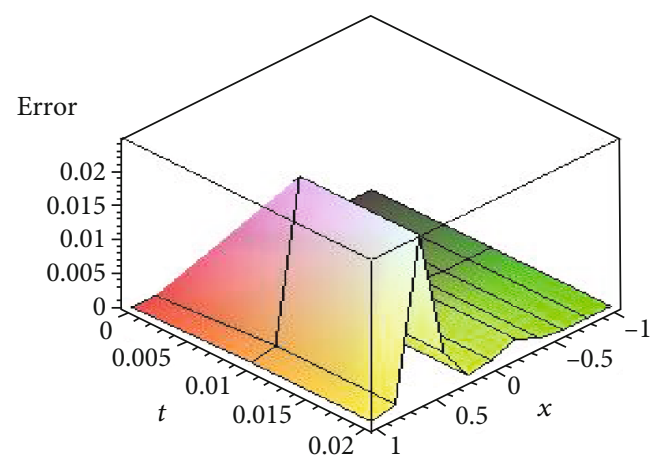

Plutonium $v=0.21$

Figure 11: The value absolute error by PNM at $N=10$ and $T=$ 0.02 .

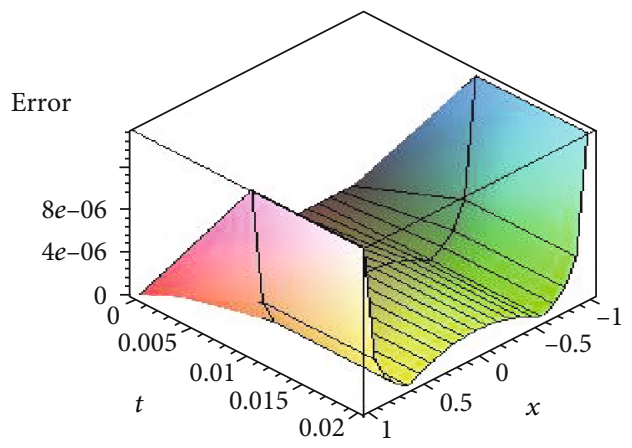

Plutonium $v=0.21$

Figure 12: The value absolute error by TMM at $N=20$ and $T=$ 0.02 .

$$
\begin{aligned}
H w & =\frac{\lambda}{\eta \pi} \int_{-1}^{1} \int_{-1}^{1} p_{1}|x-u| p_{2}|y-v| w(u, v ; t) d u d v, D w \\
& =\frac{\lambda}{\eta} \int_{-1}^{1} k(x, y) w(y, t) d y, \zeta w=\frac{\lambda}{\eta} \int_{0}^{t} \zeta(t, \tau) w(x, \tau) d \tau
\end{aligned}
$$

We assume the following conditions:

(1) The singular kernel of FI term satisfies in $L_{2}[-1,1]$ $\times L_{2}[-1,1]$ the discontinuity condition

$$
\left[\int_{-1}^{1} \int_{-1}^{1} \int_{-1}^{1} \int_{-1}^{1}\left\|p_{1}|x-u| p_{2}|y-v|\right\|\right]^{2} d x d u d t d v^{\frac{1}{2}}=M,(M \text { is a constant }) .
$$

(2) The kernel of VI term $\zeta(t, \tau)$ is continuous in the Banach space $C[0, T], 0 \leq \tau \leq t \leq T<\infty$ and satisfies

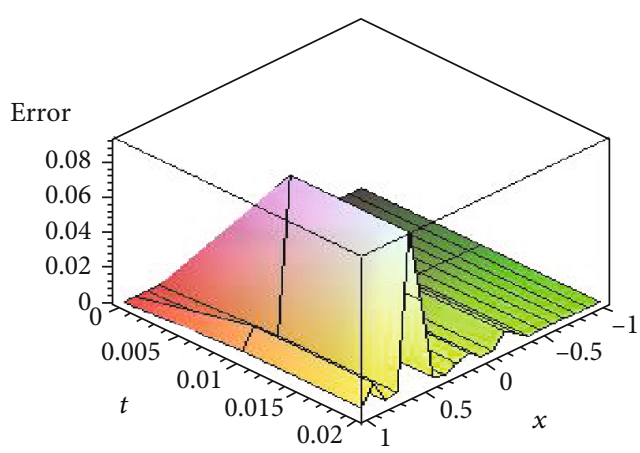

Plutonium $v=0.21$

Figure 13: The value absolute error by PNM at $N=20$ and $T=$ 0.02 .

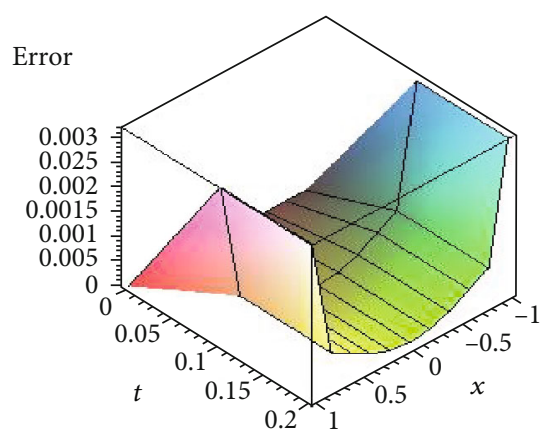

Plutonium $v=0.21$

FIgure 14: The value absolute error by TMM at $N=10$ and $T=0.2$

$$
|\zeta(t, \tau) \leq N, \quad \forall t, \tau \in[0, T]|
$$

(3) The continuous kernel $|k(x, y)| \leq L$

$$
\|f(x, t)\|=\max _{0 \leq t \leq T} \int_{0}^{t}\left[\int_{-1}^{1} f^{2}(x, \tau) d x\right]^{\frac{1}{2}} d \tau=R, \quad(R \text { is a constant }) .
$$

(4) $w(x, t)$ in the space, $L_{2}[-1,1] \times L_{2}[-1,1] \times C[0, T]$, behaves as the known function $f(x, t)$

Theorem 1. Eq. (34) has an exact unique solution in $L_{2}[-1$ $, 1] \times L_{2}[-1,1] \times C[0, T]$, under the condition

$$
|\eta|>|\lambda|\left(\frac{M}{\pi}+\sqrt{2} T(L+N)\right), T=\max _{0 \leq t \leq T} t .
$$

Lemma 1. The integral operator $\bar{W}$ maps $L_{2}[-1,1] \times L_{2}[-1$, $1] \times C[0, T]$ into itself. 


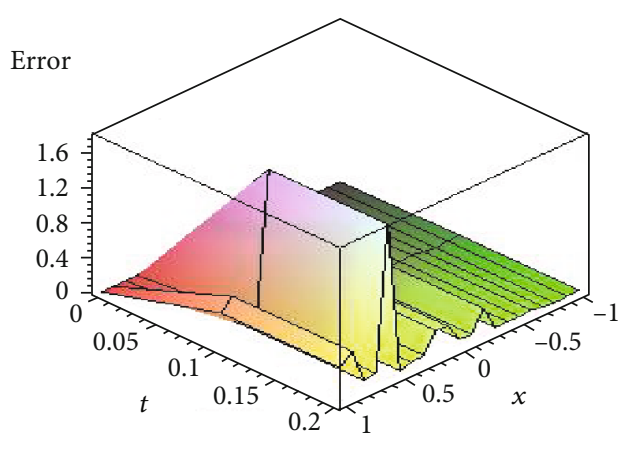

Plutonium $v=0.21$

FIgURE 15: The value absolute error by PNM at $N=10$ and $T=0.2$.

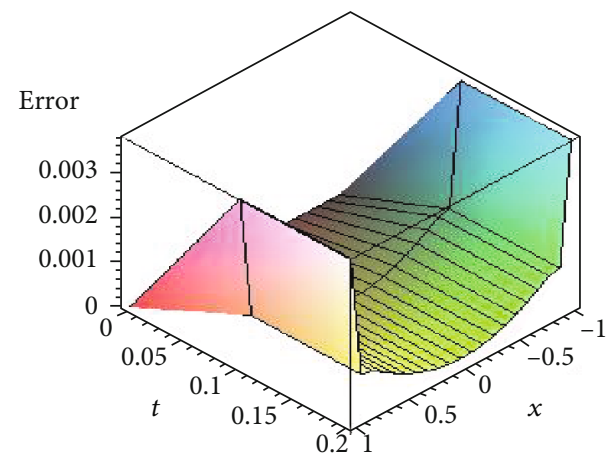

Plutonium $v=0.21$

FIgUre 16: The value absolute error by TMM at $N=20$ and $T=0.2$

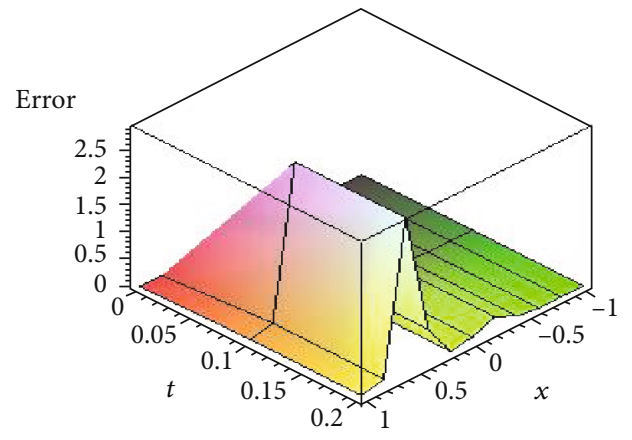

Plutonium $v=0.21$

Figure 17: The value absolute error by PNM at $N=20$ and $T=0.2$.

Proof. From (35) and (40), the normality of the integral operator takes the forms

$$
\begin{aligned}
\|\bar{W} w(x, t)\| \leq & \left|\frac{1}{\eta}\right|\|f(x, t)\|+\left|\frac{\lambda}{\pi \eta}\right|\left\|\int_{-1}^{1} \int_{-1}^{1} p_{1}|x-u| p_{2}|y-v| w(u, v ; t) d u d v\right\| \\
& +\left|\frac{\lambda}{\eta}\left\|\int_{-1}^{1}\left|k(x, y)\left\|w(y, t)|d y|||+\left|\frac{\lambda}{\eta}\right|\right\| \int_{0}^{t}\right| \zeta(t, \tau)\right\| w(x, \tau)\right| d \tau \| .
\end{aligned}
$$

Applying Cauchy-Schwarz inequality, we have

$\|H w\| \leq\left|\frac{\lambda}{\pi \eta}\right|\left\|\left(\int_{-1}^{1} \int_{-1}^{1} p_{1}^{2}|x-u| p_{2}^{2}|y-v| d u d v\right)^{\frac{1}{2}}\left(\int_{-1}^{1} \int_{-1}^{1} w^{2}(u, v ; t) d u d v\right)^{\frac{1}{2}}\right\|$.

Using the definition of the norm in the space $L_{2}[-1,1]$ $\times L_{2}[-1,1] \times C[0, T]$, we get

$$
\begin{aligned}
\|H w\| \leq\left|\frac{\lambda}{\pi \eta}\right|\left[\int_{-1}^{1} \int_{-1}^{1}\left(\int_{-1}^{1} \int_{-1}^{1} p_{1}^{2}|x-u| p_{2}^{2}|y-v| d u d v\right) d u d v\right]_{0 \leq t \leq T}^{\frac{1}{2}} \max _{0}^{t} \\
\cdot\left[\int_{-1}^{1} \int_{-1}^{1} w^{2}(u, v ; t) d u d v\right]^{\frac{1}{2}} d \tau .
\end{aligned}
$$

Then, using condition (1), we obtain

$$
\|H w\| \leq\left|\frac{\lambda}{\pi \eta}\right| M\|w\| .
$$

Also, the term $D w$ takes the form

$$
\|D w\| \leq\left|\frac{\lambda}{\eta}\right||| \int_{-1}^{1}|k(x, y) \| w(y, t)| d y|| .
$$

Using condition (3), we get

$$
\|D w\| \leq\left|\frac{\lambda}{\eta}\right| L \mid \max _{0 \leq t \leq T} \int_{0}^{t}\left[\int_{-1}^{1} w^{2}(y, \tau) \mid d y^{\frac{1}{2}} d \tau\right] \| .
$$

Hence,

$$
\|D w\| \leq\left|\frac{\lambda}{\eta}\right| \sqrt{2} L T\|w\| .
$$

In the same manner, we can write

$$
\|\zeta w\| \leq\left|\frac{\lambda}{\eta}\right|\left\|\int_{0}^{t}|\zeta(t, \tau) \| w(x, \tau)| d \tau|| .\right.
$$

Using condition (2), we obtain

$$
\|\zeta w\| \leq\left|\frac{\lambda}{\eta}\right| N\left\|\max _{0 \leq t \leq T} \int_{0}^{t}\left(\int_{-1}^{1} w^{2}(y, \tau) d y\right)^{\frac{1}{2}} d \tau\right\| .
$$

Thus, one has

$$
\|\zeta w\| \leq\left|\frac{\lambda}{\eta}\right| \sqrt{2} N T\|w\|, \quad T=\max _{0 \leq t \leq T} \mathrm{t} .
$$

Hence, with the aid of conditions (5), (44), (47), and 
(50), Eq. (41) takes the form

$$
\|\bar{W} w\| \leq \frac{R}{|\eta|}+\alpha\|w\|, \quad\left(\alpha=\left|\eta^{-1} \lambda\right|\left(\frac{M}{\pi}+\sqrt{2} T(L+N), \quad T=\max _{0 \leq t \leq T} t .\right.\right.
$$

The inequality (51) involves the boundedness of the operators $W$ and $\bar{W}$

Lemma 2. The integral operator (35) under the condition (40) is continuous and contraction operator.

Proof. For the functions $w_{1}(x, t), w_{2}(x, t)$ in the space $L_{2}[-$ $1,1] \times L_{2}[-1,1] \times C[0, T]$, formula $(35)$ yields

$$
\left\|\left(\bar{W} w_{1}-\bar{W} w_{2}\right)(x, t)\right\|=\left\|W\left(w_{1}-w_{2}\right)(x, t)\right\| .
$$

Hence, we have

$$
\begin{aligned}
\left\|\left(\bar{W} w_{1}-\bar{W} w_{2}\right)(x, t)\right\| \leq & \left|\frac{\lambda}{\eta \pi}\right|\left\|\int_{-1}^{1} \int_{-1}^{1}\left|p_{1}\right| x-u\left|p_{2}\right| y-v\left|\| w_{1}(u, v ; t)-w_{2}(u, v ; t)\right| d u d v||\right. \\
& +\left|\frac{\lambda}{\eta \pi}\right|\left\|\int_{-1}^{1}|k(|x, y|)| w_{1}(y, t)-w_{2}(y, t) \mid d y\right\| \\
& +\left|\frac{\lambda}{\eta}\right|\left\|\int_{0}^{t}|\zeta(t, \tau)| w_{1}(x, \tau)-w_{2}(x, \tau) \mid d \tau\right\| .
\end{aligned}
$$

Using formula (53) with the conditions (1), (2), and (3), then applying Cauchy-Schwarz inequality, we obtain

$$
\left\|\bar{W} w_{1}-\bar{W} w_{2}\right\| \leq \alpha\left\|w_{1}-w_{2}\right\|, \quad\left(\alpha=\left|\eta^{-1} \lambda\right|\left(\frac{M}{\pi}+\sqrt{2} T(L+N)\right)\right) .
$$

Hence, $\bar{W}$ is a continuous operator in the space $L_{2}[-1$, 1] $\times L_{2}[-1,1] \times C[0, T]$, and under the condition $(\alpha<1), \bar{W}$ is a contraction operator.

\section{THE STDFIES}

Consider Eq. (34). In this section, we divide the interval $[0$ , T], $0 \leq t \leq T<\infty, 0=t_{0}<t_{1}<\cdots<t_{n}=T$, where $t=t_{i}, i=0,1$ $, 2, . ., n$, , to get

$$
\begin{aligned}
& \eta w\left(x, t_{i}\right)+\frac{\lambda}{\pi} \int_{-1}^{1} \int_{-1}^{1} \frac{w\left(u, v, t_{i}\right)}{(x-u)(y-v)} d u d v+\lambda \int_{-1}^{1} k(x, y) w\left(y, t_{i}\right) d y \\
& +\lambda \int_{0}^{t_{i}} \zeta\left(t_{i}, \tau\right) w(x, \tau) d \tau=f\left(x, t_{i}\right) .
\end{aligned}
$$
becomes

Using the quadrature formula, the Volterra term

$$
\int_{0}^{t_{i}} \zeta\left(t_{i}, \tau\right) w(x, \tau) d \tau=\sum_{j=0}^{i} \kappa_{j} \zeta\left(t_{i}, t_{j}\right) w\left(x, t_{j}\right)+R\left(x, t_{i}\right)
$$

$\kappa_{j}$ is the weight, where $\kappa_{0}=1 / 2 h_{0}, \kappa_{i}=1 / 2 h_{i}, \kappa_{i}=h_{j}, 0<$ $j<i, h$ denotes the constant step size for integration. Using
(56) in (55), we have

$$
\begin{aligned}
& \eta w_{i}(x)+\frac{\lambda}{\pi} \int_{-1}^{1} \int_{-1}^{1} \frac{w_{i}(u, v)}{(x-u)(y-v)} d u d v+\lambda \int_{-1}^{1} k(x, y) w_{i}(y) d y+\lambda \sum_{j=0}^{i} \kappa_{j} \zeta_{i, j} w_{j}(x) \\
& =f_{i}(x)+R_{i}(x) .
\end{aligned}
$$

Formula (57) can be adapted in the form

$$
\begin{aligned}
\left(\eta+\lambda \kappa_{i} \zeta_{i, j}\right) w_{i}(x)= & f_{i}(x)-\frac{\lambda}{\pi} \int_{-1}^{1} \int_{-1}^{1} p_{1}(|x-u|) p_{2}(|y-v|) w_{i}(u, v) d u d v \\
& -\lambda \int_{-1}^{1} k(x, y) w_{i}(y) d y-\lambda \sum_{j=0}^{i} \kappa_{j} \zeta_{i, j} w_{j}(x), 0 \leq j \leq i-1 .
\end{aligned}
$$

Then, the general form of Eq. (58) can be represented as

$$
\begin{aligned}
& \mu_{i} w_{i}(x)+\frac{\lambda}{\pi} \int_{-1}^{1} \int_{-1}^{1} p_{1}(|x-u|) p_{2}(|y-v|) w_{i}(u, v) d u d v \\
& +\lambda \int_{-1}^{1} k(x, y) w_{i}(y) d y=\psi_{i}(x)
\end{aligned}
$$

where

$$
\mu_{i}=\left(\eta+\lambda \kappa_{i} \zeta_{i, j}\right), \quad \psi_{i}=f_{i}(x)-\lambda \sum_{j=0}^{i-1} \kappa_{j}\left[\zeta_{i, j} w_{j}(x)\right], \quad i=0,1, \cdots, n
$$

Formula (59) represents a linear system of TDFIEs of the second kind, which contains $(n+1)$ equation of $(n+1)$ unknown functions of $w_{i}(x)$ corresponding to the time interval $[0, T]$.

\section{Some Numerical Methods}

5.1. The TMM. We present the TMM to obtain numerical solution of TDFIE of the second kind with Cauchy form, which it expresses in the form

$$
\begin{aligned}
& \mu_{i} w_{i}(x)+\frac{\lambda}{\pi} \int_{-1}^{1} \int_{-1}^{1} p_{1}(|x-u|) p_{2}(|y-v|) w_{i}(u, v) d u d v \\
& +\lambda \int_{-1}^{1} k(x, y) w_{i}(y) d y=\psi_{i}(x)
\end{aligned}
$$

which it may be adapted as

$$
\mu w(x)=\psi(x)-\frac{\lambda}{\pi} \int_{-1}^{1} \int_{-1}^{1} F(x-u, y-v) w(u, v ; t) d u d v
$$


where

$F(x-u, y-v)=\frac{(1+\pi k(x, y)(y-x))}{(x-u)(y-v)}=\left(\frac{1}{(x-u)(y-v)}\right)(1+\pi k(x, y)(y-x))$.

Then, write the integral term in Eq. (62) as the form

$$
\begin{aligned}
& \int_{-1}^{1} \int_{-1}^{1} F(x-u, y-v) w(u, v ; t) d u d v \\
& \quad=\sum_{n=-N}^{N} \int_{n h}^{n h+h} F(x-u, y-v) w(u, v ; t) d u d v,\left(h=\frac{1}{N}\right) .
\end{aligned}
$$

Formula (64) reduces as

$$
\begin{aligned}
& \int_{-1}^{1} \int_{-1}^{1} F(x-u, y-v) w(u, v ; t) d u d v \\
& \quad=\sum_{n=-N}^{N} \sum_{m=-M}^{M} F(x-u, y-v) w(u, v ; t) d u d v\left(h=\frac{1}{N}\right) .
\end{aligned}
$$

Then,

$$
\begin{aligned}
\int_{n h}^{n h+h} & \int_{m h}^{m h+h} F(x-u, y-v) w(u, v ; t) d u d v \\
\quad= & A_{n, m}(x, y) w(n h, m h)+B_{n, m}(x, y) w(n h+h, m h+h)+R .
\end{aligned}
$$
obtain

Then, we put $w(u, v)=1.1, u v$ in Eq. (66), and then we

$$
\begin{gathered}
A_{n, m}(x, y)=\frac{1}{h}\left[\frac{(n h+h)(m h+h) I}{(n h+m h+h)}-\frac{J}{(n h+m h+h)}\right], \\
B_{n, m}(x, y)=\frac{1}{h}\left[\frac{J}{(n h+m h+h)}-\frac{(n h)(m h) I}{(n h+m h+h)}\right],
\end{gathered}
$$

where

$$
\begin{gathered}
I(x, y)=\int_{n h}^{n h+h} \int_{m h}^{m h+h} k(|t-\tau|,|x-y|) d u d v, \\
J(x, y)=\int_{n h}^{n h+h} \int_{m h}^{m h+h} u v \cdot k(|x-u|,|y-v|) d u d v .
\end{gathered}
$$

Eq. (65) becomes

$$
\begin{aligned}
& \int_{-1}^{1} \int_{-1}^{1} F(x-u, y-v) w(u, v ; t) d u d v \\
& \quad=\sum_{n=-N}^{N-1} \sum_{m=-M}^{M-1}\left[A_{n, m}(x, y) w(n h, m h ; t)+B_{n, m}(x, y) w(n h+h, m h+h ; t)\right] \\
& \quad=\sum_{n=-N}^{N-1} \sum_{m=-M}^{M-1} A_{n, m}(x, y) w(n h, m h ; t)+\sum_{n=-N}^{N} \sum_{m=-M}^{M} B_{(n-1)(m-1)}(x, y) w(n h, m h ; t) \\
& \quad=\sum_{n=-N}^{N} \sum_{m=-M}^{M} D_{n, m}(x, y) w(n h, m h ; t),
\end{aligned}
$$

where

$$
D_{n, m}(x, y)= \begin{cases}A_{-N}(x, y) & n=m=-N, \\ A_{n}(x, y)+B_{n-1}(x, y) & -N<n=m<N, \\ B_{N-1}(x, y) & n=m=N .\end{cases}
$$

Thus, the IE (62) becomes

$$
\mu w(x, y)+\frac{\lambda}{\pi} \sum_{n=-N}^{N} \sum_{m=-M}^{M} D_{n, m}(x, y) w(n h, m h ; t)=f(x, y) .
$$

If we put $x=k h, y=l h$, then we get

$$
\mu w_{k, l}+\frac{\lambda}{\pi} \sum_{n=-N}^{N} \sum_{m=-M}^{M} D_{k l n, m} w_{n m}=f_{k l} \quad-N \leq k \leq N,-M \leq l \leq M,
$$

where

$$
D_{k l n, m}= \begin{cases}A_{-N}(k h, l h) & n=m=-N, \\ A_{n}(k h, l h)+B_{n-1}(k h, l h) & -N<n=m<N, \\ B_{N-1}(k h, l h) & n=m=N .\end{cases}
$$

The matrix $D_{k l n, m}$ may be written as $D_{k l n, m}=G_{k l n, m}-$ $E_{k l n, m}$, where

$$
G_{k l n, m}=A_{n}(k h, l h)+B_{n-1}(k h, l h), \quad-N \leq k, l, n, \leq N
$$

is the TM of order $2 N+1$, and the matrix

$$
E_{k l n, m}= \begin{cases}B_{-N-1}(k h, l h) & n=m=-N, \\ 0 & -N<n=m<N, \\ A_{N}(k h, l h) & n=m=N .\end{cases}
$$

However, the solution of the system can be obtained in 
the form

$$
w_{k, l}=\left[\mu I-\lambda\left(G_{k l n}-E_{k l n}\right)\right]^{-1} f_{k l},
$$

where $I$ is the identity matrix and $\left|\mu I-\lambda\left(G_{k l n}-E_{k l n}\right)\right| \neq 0$..

\subsection{The PNM. Consider}

$$
\mu w(x)=\psi(x)-\frac{\lambda}{\pi} \int_{-1}^{1} \int_{-1}^{1} p(x-u ; y-v) \bar{k}(|x-u| ;|y-v|) w(u, v ; t) d u d v
$$

where

$$
p(x-u ; y-v) \bar{k}(|x-u| ;|y-v|)=\left(\frac{1}{(x-u)(y-v)}\right)(1+\pi k(x, y)(y-x)),
$$

where pand $\bar{k}$ are badly behaved and well-behaved functions of their arguments, respectively. We approximate the integral term in (77) when $\left\{\psi_{s}\left(x_{i}, y_{i}\right)\right\}$ by

$$
\begin{aligned}
& \int_{-1}^{1} \int_{-1}^{1} p\left(x_{i}-u ; y_{s}-v\right) \bar{k}\left(\left|x_{i}-u\right| ;\left|y_{s}-v\right|\right) w(u, v ; t) d u d v \\
& \quad \approx \sum_{j=0}^{N} \sum_{i=0}^{M} \kappa_{i j s l} \bar{k}\left(\left|x_{i}-u\right| ;\left|y_{s}-v\right|\right) w\left(u_{j}, v_{l}\right),
\end{aligned}
$$

where $\kappa_{i j s l}$ is the weights. Also, we approximate the integral term in (77) in the form:

$$
\begin{aligned}
& \int_{-1}^{1} \int_{-1}^{1} p\left(x_{i}-u ; y_{s}-v\right) \bar{k}\left(\left|x_{i}-u\right| ;\left|y_{s}-v\right|\right) w(u, v ; t) d u d v \\
& \quad=\sum_{j=0}^{N} \sum_{i=0}^{M} \int_{u_{2 j}}^{u_{2 j+2}} \int_{v_{2 j}}^{v_{2 j+1}} p\left(x_{i}-u ; y_{s}-v\right) \bar{k}\left(\left|x_{i}-u\right| ;\left|y_{s}-v\right|\right) w(u, v ; t),
\end{aligned}
$$

where $x_{i}=u_{i}=y_{i}=v_{i}=a+i h, i=0,1, \cdots, N$ with $h=b$ $-a / N$ and $N$ even. Now, if we approximate the nonsingular part of the integrand over each interval $\left[u_{2 j}, u_{2 j+2}\right],\left[\mathrm{V}_{21}, v_{2 l+2}\right]$ , by the second degree Lagrange interpolation polynomial that interpolates, we find

$$
\begin{aligned}
& \int_{-1}^{1} \int_{-1}^{1} p\left(u_{i}-u, v_{i}-v\right) \tilde{k}\left(u_{i}-u, v_{i}-v\right) w(u, v ; t) d u d v=\sum_{j=0}^{\frac{N-2}{2}} \sum_{l=0}^{\frac{M-2}{2}} \int_{u_{2 j}}^{u_{2 j+2}} \int_{v_{2 l}}^{v_{2 l+2}} p\left(u_{i}-u, v_{i}-v\right) \\
& \quad \times\left\{\frac{\left(u_{2 j+1}-u\right)\left(v_{2 l+1}-v\right)\left(u_{2 j+2}-u\right)\left(v_{2 l+2}-v\right)}{\left(2 h^{2}\right)\left(2 h^{2}\right)} \tilde{k}\left(u_{i}-u_{2 j}, v_{i}-v_{2 l}\right) w\left(u_{2 j}, v_{2 l} ; t\right)\right. \\
& \quad+\frac{\left(u-u_{2 j}\right)\left(v-v_{2 l}\right)\left(u_{2 j+2}-u\right)\left(v_{2 l+2}-v\right)}{\left(h^{2}\right)\left(h^{2}\right)} \tilde{k}\left(u_{i}-u_{2 j+1}, v_{i}-v_{2 l+1}\right) w\left(u_{2 j+1}, v_{2 l+1} ; t\right) \\
& \left.\quad+\frac{\left(u-u_{2 j}\right)\left(v-v_{2 l}\right)\left(u-u_{2 j+1}\right)\left(v-v_{2 l+1}\right)}{\left(2 h^{2}\right)\left(2 h^{2}\right)} \tilde{k}\left(u_{i}-u_{2 j+2}, v_{i}-v_{2 l+2}\right) w\left(u_{2 j+2}, v_{2 l+2} ; t\right)\right\} d u d v \\
& =\sum_{j=0}^{N} \sum_{l=0}^{M} w_{i j} w_{i} \tilde{k}\left(u_{i}-u_{j}, v_{i}-v_{l}\right) w\left(u_{i}, v_{l} ; t\right),
\end{aligned}
$$

where $u_{j}=j h, u_{j+1}=(j+1) h, u_{j}-u_{j+1}=v_{l}-v_{l+1}=-h$.
If we define

$$
\begin{aligned}
\alpha_{i j}\left(u_{i}, v_{s}\right)= & \frac{1}{4 h^{4}} \int_{u_{2 j-2}}^{u_{2 j}} \int_{v_{2 j-2}}^{v_{2 j}} p\left(u_{i}, u ; v_{s}, v\right)\left(u-u_{2 j-2}\right)\left(v-v_{2 j-2}\right) \\
& \cdot\left(u-u_{2 j-1}\right)\left(v-v_{2 j-1}\right) d u d v,
\end{aligned}
$$

$$
\begin{aligned}
\beta_{i j}\left(u_{i}, v_{s}\right)= & \frac{1}{4 h^{4}} \int_{u_{2 j-2}}^{u_{2 j}} \int_{v_{2 j-2}}^{v_{2 j}} p\left(u_{i}, u ; v_{s}, v\right)\left(u_{2 j-2}-u\right)\left(v_{2 j-2}-v\right) \\
& \cdot\left(u_{2 j}-u\right)\left(v_{2 j}-v\right) d u d v
\end{aligned}
$$

$$
\begin{aligned}
\gamma_{i j}\left(u_{i}, v_{s}\right)= & \frac{1}{4 h^{4}} \int_{u_{2 j-2}}^{u_{2 j}} \int_{v_{2 j-2}}^{v_{2 j}} p\left(u_{i}, u ; v_{s}, v\right)\left(u-u_{2 j-2}\right)\left(v-v_{2 j-2}\right) \\
& \cdot\left(u-u_{2 j-1}\right)\left(v-v_{2 j-1}\right) d u d v .
\end{aligned}
$$

In general, assume $K_{0}$ thus (82) become

$$
\begin{aligned}
\alpha_{i j}\left(u_{i}, v_{s}\right)= & \frac{h^{2}}{4} \int_{0}^{2} \int_{0}^{2} \zeta \delta(\zeta-1)(\zeta-1) p\left(u_{i},\left(u_{2 j-2}+\zeta h\right) ; v_{s},\left(v_{2 l-2}+\delta h\right)\right) d \zeta d \delta, \\
\beta_{i j}\left(u_{i}, v_{s}\right)= & \frac{h^{2}}{4} \int_{0}^{2} \int_{0}^{2}(\zeta-1)(\zeta-2)(\zeta-1)(\zeta-2) p\left(u_{i},\left(u_{2 j-2}+\zeta h\right) ; v_{s},\right. \\
& \left.\cdot\left(v_{2 l-2}+\delta h\right)\right) d \zeta d \delta, \\
\gamma_{i j}\left(u_{i}, v_{s}\right)= & \frac{h^{2}}{4} \int_{0}^{2} \int_{0}^{2} \zeta \delta(2-\zeta)(2-\zeta) p\left(u_{i},\left(u_{2 j-2}+\zeta h\right) ; v_{s},\left(v_{2 l-2}+\delta h\right)\right) d \zeta d \delta .
\end{aligned}
$$

If we define $\psi_{k}=\int_{0}^{2} \int_{0}^{2} \xi^{k} \delta^{k} p\left(u_{i}-\left(u_{2 j-2}+\xi h\right), v_{i}-\left(v_{2 l-2}\right.\right.$ $+\delta h)) d \xi d \delta, k=0,1,2$ and let $u_{i}-u_{2 j-2}=(i-2 j+2) h, v_{i}-$ $v_{2 l-2}=(i-2 l+2) h$, we have $\psi_{k}=\int_{0}^{2} \int_{0}^{2} \xi^{k} \delta^{k} p(\mid z-\zeta) h||,(g-$ $\delta) h \mid) d \zeta d \delta, k=0,1,2, z=i-2 j+2, \mathrm{~g}=s-2 l+2$.

\section{Numerical Applications and Discussions}

In this section, we state some applications and numerical results to discuss the approximate solution (i.e., the treat of the surface cracks that occurs in the materials as a result, the constant in finite of materials, which the deformation increases as well as the time increases in the interval $[0, T]$. The TMM and PNM are used to get numerical solution for values of $\mu=1$, and for different materials, plutonium $v=$ 0.21 and fiber $v=0.22$, where the Poisson ratio is $0 \leq v<1$ $, \lambda=\bar{\lambda} / 2 G, \bar{\lambda}=2 G v /(1-2 v),(G$ shear modules $)$.We divided the position interval by $N=10,20$ units. Since $0 \leq t \leq T<\infty$ , we choose the time $T=0.02,0.2$. Tables 1 and 2 are as follows: exact sol. $\rightarrow$ the exact solution, Approx.T. $\rightarrow$ approximate solution of TMM, error.T. $\rightarrow$ the absolute error of TMM, Approx.N. $\rightarrow$ approximate solution of PNM, and error.N. $\rightarrow$ the absolute error of PNM. Figures $2-17$ show the value absolute error by TMM and PNM at $N=10,20$ and the time $T=0.02,0.2$, for the materials plutonium $v=$ 0.21 and fiber $v=0.22$. 
Consider

$$
\begin{aligned}
\mu w(x, t)= & f(x, t)-\frac{\lambda}{\mu} \int_{-1}^{1} \int_{-1}^{1} \frac{1}{(x-u)(y-v)} w(u, v ; t) d u d v \\
& -\lambda \int_{-1}^{1}|x-y| w(y, t) d y-\int_{0}^{t} t \tau w(x, \tau) d \tau,(\mu=1) .
\end{aligned}
$$

The exact solution $\phi(x, y)=x^{2} y^{2}$.

\section{The Conclusion}

We have presented a successful technique for the numerical solution of MIE with singular kernel in two-dimensional by using TMM and PNM which is established from the plane strain problem for the bounded layer medium composed of different materials. From Tables 1 and 2 and Figures 2-17, we note that the errors due to the TMM are less than the errors due to PNM. In addition, we note that $N$ increases for the two different materials (fiber $v=0.22$ ) (plutonium $v$ $=0.21)$, the values of $k(|g(x)-g(y)|)=\cot (g(y)-g(x) / 2)$ and $x \in[-\pi, \pi], \phi( \pm \pi, \mathrm{t})=0$. are fixed, and the error values increase. The approximate solution is nearly coincident with the exact solution for $t>0$ at each value of $x \in[-1,1]$.

\section{Data Availability}

All the data are available within the article and also as the references.

\section{Conflicts of Interest}

The author declares that he/she has no conflicts of interest.

\section{References}

[1] H. Brunner and J. P. Kauthen, "The numerical solution of twodimensional volterra integral equations by collocation and iterated collocation," IMA Journal of Numerical Analysis, vol. 9, no. 1, pp. 47-59, 1989.

[2] B. A. Beltyukov and L. N. Kuznechikhina, "A runge-kutta method for the solution of two-dimensional nonlinear volterra integral equations," Differential Equations, vol. 12, pp. 11691173, 1976.

[3] P. Darania and A. Ebadian, "Numerical solutions of the nonlinear two-dimensional volterra integral equations," NJOM, vol. 36, 2007.

[4] M. A. Abdou, "Fredholm-Volterra integral equation of the first kind and contact problem," Applied Mathematics and Computation, vol. 125, no. 2-3, pp. 177-193, 2002.

[5] M. A. Abdou, "Fredholm-volterra integral equation with singular kernel," Applied Mathematics and Computation, vol. 137, no. 2-3, pp. 231-243, 2003.

[6] M. M. EL-Borai, M. A. Abdou, and M. Basseem, "An analysis of two-dimensional integral equations of the second kind," LEMATHEMATICHE LXII, vol. 15, 2007.

[7] A. M. AL-Bugami, "Two-dimensional singular fredholm integral equation with applications in contact problems. Jordan," Journal of Mathematics and Statistics, vol. 4, 2011.
[8] A. M. AL-Bugami, "two dimensional Fredholm integral equation with time," Journal of Modern Methods in Numerical Mathematics, vol. 3, no. 2, pp. 66-78, 2012.

[9] F. Erdogan, G. D. Gupta, and T. S. Cook, "Numerical solution of singular integral equations," in In: Mechanics of fracture, G. C. Sih, Ed., vol. 1, pp. 368-425, Methods of analysis and solutions of crack problems, Noordhoff, Leyden. The Netherland. Chap. 7, 1973.

[10] A. M. Al-Bugami and M. M. Al-Wagdani, "Runge-Kutta and block by block methods to solve linear two-dimensional Volterra integral equation with continuous kernel," Journal of Advances In Mathematics, vol. 11, no. 10, pp. 5705-5714, 2016.

[11] A. M. Al-Bugami, "Some techniques for solving FredholmVolterra integral equation of the second kind," IJRRAS, vol. 40, no. 1, pp. 40-51, 2019.

[12] A. M. Al-Bugami, "Singular Hammerstein-Volterra integral equation and its numerical processing," Journal of Applied Mathematics and Physics, vol. 9, no. 2, pp. 379-390, 2021.

[13] H. Khayyat, "On treating of surface cracks in finite layers of fractional materials," American academic scientific research journal for engineering, technology and sciences, vol. 34, no. 1, pp. 213-230, 2017. 